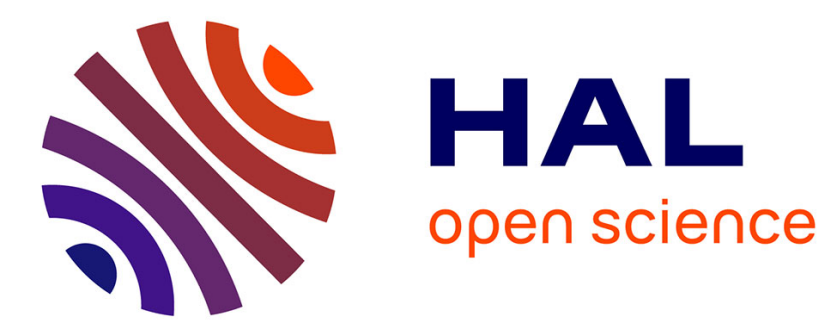

\title{
Le cofinancement usager - contribuable et le partenariat public - privé changent les termes de l'évaluation des programmes d'investissement public
}

William Roy, Alain Bonnafous, Pablo Jensen

\section{- To cite this version:}

William Roy, Alain Bonnafous, Pablo Jensen. Le cofinancement usager - contribuable et le partenariat public - privé changent les termes de l'évaluation des programmes d'investissement public. Economie et Prévision, 2006, 175-176 (2006/4-5), 23 p. 10.3917/ecop.175.0015 . halshs-00122918

\section{HAL Id: halshs-00122918 \\ https://shs.hal.science/halshs-00122918}

Submitted on 5 Jan 2007

HAL is a multi-disciplinary open access archive for the deposit and dissemination of scientific research documents, whether they are published or not. The documents may come from teaching and research institutions in France or abroad, or from public or private research centers.
L'archive ouverte pluridisciplinaire HAL, est destinée au dépôt et à la diffusion de documents scientifiques de niveau recherche, publiés ou non, émanant des établissements d'enseignement et de recherche français ou étrangers, des laboratoires publics ou privés. 


\title{
Le cofinancement usager - contribuable et le partenariat public - privé changent les termes de l'évaluation des programmes d'investissement public
}

\author{
Alain Bonnafous ${ }^{\mathrm{a}}$, Pablo Jensen ${ }^{\mathrm{a}, \mathrm{b}}$ et William Roy ${ }^{\mathrm{a}}$ \\ a- Laboratoire d'Économie des Transports, Lyon, France. \\ b- Laboratoire de la Physique de la Matière Condensée et des Nanostructures, Villeurbanne, France. \\ (alain.bonnafous@let.ish-lyon.cnrs.fr,pablo.jensen@let.ish-lyon.cnrs.fr,william.roy@let.ish-lyon.cnrs.fr)
}

\begin{abstract}
Résumé : Bien qu'il puisse être appliqué à diverses sortes d'équipements publics, l'objet de cet article concerne les investissements d'infrastructure dans le secteur des transports. Il pose le problème de l'articulation entre les évaluations économiques et financières. Il s'agit d'optimiser le rendement social au sens du calcul économique, mais en prenant en compte deux aspects trop négligés : d'une part, le fait que la plupart des grands investissements candidats seront cofinancés par l'usager et le contribuable, comme l'ont été les investissements récents; d'autre part, la nécessité de ne pas seulement raisonner sur la détermination du meilleur projet, mais sur la détermination du meilleur programme de projets. Cet article montre l'attrait du ratio de "l'utilité collective par euro public investi », en tant que critère permettant appréhender les effets de l'intensification de la contrainte financière publique.
\end{abstract}

Mots clés : Infrastructure, investissement, ordre, choix public.

Classification JEL : D6 ; D7 ; H54 ; L9 ; R42

\section{Position du problème}

Les investissements les plus significatifs des dernières décennies répondaient, en principe, à une double logique. Une logique d'utilité collective et une logique de financement par l'usager. Certaines décision d'investir s'inscrivent strictement dans la première (soussection 1.1). D'autres relèvent strictement de la logique de financement par l'usager (soussection 1.2). Cependant, aujourd'hui, la plupart des nouvelles infrastructures de transport impliquent une logique mixte (sous-section 1.3).

\subsection{La logique de l'utilité collective}

Les deux modalités de financement, par la puissance publique ou par l'usager, ont été initialement considérées comme exclusives l'une de l'autre. La première concerne les infrastructures sans péage et renvoie à la tradition du bien public pur. Le cas des routes en est un bon exemple: même si l'on peut considérer que des taxes diverses (accises, taxes à l'essieu ou vignette) constituent une tarification d'usage des infrastructures, ces ressources s'inscrivent dans "l'unité de la recette » qui dérive de la tradition des finances publiques. Il n'y a pas de ressource affectée et c'est le budget général (national ou territorial) qui finance ces infrastructures. 
La décision d'investir renvoie alors à la logique de la rentabilité collective. Celle-ci est appréciée sur la base d'un bilan actualisé qui met en balance les richesses détruites (les coûts de toute nature) et les richesses créées (les avantages de toute nature) du fait de la réalisation du projet. L'évaluation des grands projets d'infrastructure de transport par l'analyse coûtsavantages (ACA) est éprouvée dans les pays développés (Hayashi \& Morisugi 2000). En France, la loi ${ }^{1}$ prévoit que « les choix relatifs aux infrastructures, équipements et matériels de transport et donnant lieu à financement public, en totalité ou partiellement, sont fondés sur l'efficacité économique et sociale de l'opération. »Et si la démarche n'enthousiasme pas toujours les décideurs politiques ${ }^{2}$, elle a le remarquable avantage d'éclairer les choix sur un certain nombre de dimensions (Quinet 2000).

Les bases théoriques de ce calcul économique sont connues, en particulier dans leur formulation la plus achevée (Lesourne, 1972) ) $^{3}$ Leur déclinaison pour les investissements routiers ou autoroutiers de rase campagne a été, par exemple, explicitée par des circulaires ministérielles au début des années $60^{4}$. Il s'agit d'une logique de rendement social, ou de rentabilité socio-économique, fondée sur un bilan actualisé élargi à l'ensemble des coûts et avantages pour la collectivité, y compris divers effets externes. La Valeur Actualisée Nette (VAN) socio-économique est une approximation de l'utilité sociale d'un projet. On définit classiquement la VAN socio-économique d'un projet par la formulation suivante, actualisant au taux $\alpha$ et à sa date de mise en service les avantages nets ${ }^{5} \Delta A_{t}$, et capitalisant les dépenses d'investissement $\Delta I_{t}$ :

$$
V A N=\sum_{t=-d}^{0} \frac{-\Delta I_{t}}{(1+\alpha)^{t}}+\sum_{t=1}^{n} \frac{\Delta A_{t}}{(1+\alpha)^{t}}
$$

Il en résulte un taux de rentabilité interne généralement appelé taux de rentabilité socioéconomique ou, plus simplement, économique (TRE), qui est le taux d'actualisation $\alpha$ qui équilibre les coûts et avantages actualisés du projet, c'est à dire annule la VAN. Ce n'est pas seulement le point de vue du maître d'ouvrage (ou du transporteur) et de son compte de résultat qui est retenu, mais celui de la collectivité tout entière. Les pertes et avantages de tous les agents économiques sont ainsi évalués, telles, par exemple, les pertes de recettes nettes des modes concurrents ou les variations de surplus des usagers, ou encore les conséquences du projet sur la sécurité ou l'environnement ${ }^{6}$.

Nous noterons $\mathrm{VAN}_{\mathrm{e}}$ cette valeur qui résulte du bilan actualisé socio-économique et qui est à distinguer de la valeur actualisée qui résulte du bilan financier pour l'opérateur, qui sera notée $\mathrm{VAN}_{\mathrm{f}}$ et que nous retrouverons dans la sous-section suivante. La première est calculée, en France, avec le «taux d'actualisation du plan», aujourd'hui établi à $8 \%^{7}$. Outre qu'au plan théorique cette $\mathrm{VAN}_{\mathrm{e}}$ exprime la variation d'utilité collective que provoque la réalisation du projet, elle indique si celui-ci crée dans la durée plus de richesse qu'il n'en détruit. En ce cas, elle est positive et le TRE du projet est supérieur au taux d'actualisation officiel.

Plus précisément, Abraham \& Laure (1959) montrent que la date optimale de réalisation d'un projet intervient lorsque le taux de rentabilité immédiate (rapport entre les avantages socio-économiques pour l'année de mise en service et le coût capitalisé d'investissement) atteint le niveau du taux d'actualisation. En effet, rappelons nous que " dans ces conditions, une opération isolée ne doit pas être réalisée dès qu'elle est juste rentable ${ }^{8}$, mais quelques années après seulement» (Abraham \& Laure 1959, p.744), ce qui est un résultat parfois ignoré. Si la VAN varie en fonction de la date de mise en service, un projet ne doit pas être réalisé dès que la $\mathrm{VAN}_{\mathrm{e}}$ devient positive, mais quand elle devient maximum, ce qui peut survenir plusieurs années après. 
La démonstration de ce résultat qui nous semble la plus élégante est proposée par Quinet (1998, p.237), et s'obtient en supposant une actualisation continue. A la date de mise en service $t^{*}$, on a :

$$
V A N_{e}\left(t^{*}\right)=-I . e^{-\alpha . t^{*}}+\int_{t^{*}}^{+\infty} A(t) e^{-\alpha . t} d t
$$

où $A(t)$ est une fonction des avantages nets aux dates $t$.

La condition nécessaire ${ }^{9}$ de maximisation de cette fonction est :

$$
\frac{d V A N_{e}\left(t^{*}\right)}{d t}=0 \Leftrightarrow \frac{A\left(t^{*}\right)}{I}=\alpha
$$

Le problème est donc relativement simple : dès lors que la rentabilité immédiate d'un projet a dépassé le taux d'actualisation, il faut le réaliser dès que possible. Ce critère a été largement mis en œuvre par les praticiens français ${ }^{10}$.

Toutefois, la contrainte budgétaire ne permet généralement pas de mettre en œuvre tous les projets à leur date optimale de réalisation et il y a ainsi une sorte de file d'attente de projets dont la $\mathrm{VAN}_{\mathrm{e}}$ est positive c'est à dire de projets réputés rentables. Par exemple, la France n'est actuellement pas en mesure de réunir les ressources qui seraient nécessaires pour financer l'ensemble des projets «terminant» le maillage du réseau autoroutier national. La liste des projets en attente est disponible dans le Rapport d'audit sur les grands projets d'infrastructures de transport établi par l'Inspection Générale des Finances et le Conseil Général des Ponts et Chaussées, en février 2003.

Dans la réalité, il s'agit donc de désigner le paquet de certains de ces projets qui apporte au total une $\mathrm{VAN}_{\mathrm{e}}$ maximale sous la contrainte de capacité de financement public. Les projets réalisés dans un ordre et à un rythme qui respectent cette contrainte constituent un programme. Celui-ci est optimal au sens du calcul économique s'il dégage un maximum de valeur actualisée nette globale, ce qui est assuré si les projets sont hiérarchisés selon l'ordre décroissant de la $\mathrm{VAN}_{\mathrm{e}}$ dégagée par euro investi (profitablité). Cet ordre se révèle équivalent à celui des TRE décroissants.

\subsection{La logique du financement par l'usager}

La deuxième modalité de financement a été utilisée pour les grands aéroports, pour les autoroutes à péages ou pour les premières lignes ferroviaires à grande vitesse. Pour ces projets, les recettes acquittées par les usagers étant suffisantes pour couvrir la charge de la dette nécessaire à leur financement, l'Etat confie le financement, la construction et l'exploitation à un opérateur maître d'ouvrage ou maître d'ouvrage délégué : une entreprise nationale (Aéroports de Paris, SNCF puis RFF ${ }^{11}$, par exemple), une société d'économie mixte pour les autoroutes ou, exceptionnellement, une société privée ${ }^{12}$.

Le calcul de rentabilité financière qui doit être alors mené repose sur la même logique actuarielle que celui du bilan actualisé socio-économique mais ne concerne que les éléments qui déterminent le compte de résultat de l'opérateur. A ce bilan actualisé correspond un taux de rentabilité interne, le TRI, qui est le taux d'actualisation pour lequel les sommes des charges et produits actualisés s'équilibrent.

Nous avons vu que dans la logique du financement public, le TRE pouvait être confronté à une norme officielle qui est en France le taux d'actualisation du Plan : s'il lui est supérieur le projet peut être considéré comme rentable. Le TRI se prête à une confrontation 
comparable : s'il est supérieur à un taux d'intérêt déterminé, le projet peut être considéré comme profitable.

Ce seuil de profitabilité est très variable selon les secteurs d'activité et selon la situation du marché financier. Si l'opérateur est un établissement public tenu de n'investir que si cela ne creuse pas un déficit, le seuil sera égal au taux d'intérêt de long terme du marché augmenté d'une prime de risque. Lorsque la SNCF était encore maître d'ouvrage, ce TRI minimal était établi à $8 \%$ en termes réels. Il est aujourd'hui pour RFF de $8 \%$ en termes nominaux ${ }^{13}$. Ce seuil est fixé en accord avec les autorités de tutelle. Dans le cas de sociétés privées, le seuil est de même nature mais il est augmenté d'une marge qui permet de rétribuer les fonds propres engagés. La distinction qui peut être faite entre le cas d'une entreprise publique et celui d'un partenariat public - privé (PPP) a été explorée par ailleurs (Bonnafous, 2002) et ne sera pas reprise ici. Nous considérerons dans ce qui suit que ce TRI minimal est déterminé tout à la fois par le dispositif institutionnel et par le marché et qu'il est connu.

On se trouve dans une logique stricte de financement par l'usager lorsque le TRI d'un projet est supérieur à ce TRI minimal. En ce cas, la puissance publique reste cependant maîtresse des programmes c'est à dire de l'ordre et du rythme de réalisation des projets. La raison économique d'Etat a consisté généralement à privilégier l'ordre des TRE décroissants et à ne considérer le TRI du projet qu'au titre d'une condition permissive.

En ce cas, en effet, le calcul de la rentabilité financière des projets n'a d'autre objet que de vérifier que la valeur actualisée nette financière de l'opération, $\mathrm{VAN}_{\mathrm{f}}$, soit positive. En somme, que la rentabilité financière du projet soit suffisante pour attirer un opérateur privé ou pour que l'opération puisse être lancée sans compromettre dans la durée les comptes de résultat d'un opérateur public. Encore que ce principe ait pu être contourné pour les autoroutes par le système dit d'adossement. Ce dispositif consistait à confier au concessionnaire d'un large réseau d'autoroutes la construction et l'exploitation d'une section complémentaire de ce réseau. Le financement de cette nouvelle section, en principe moins rentable que les précédentes, était d'abord assuré par les cash-flows des anciennes sections et, ensuite, par un emprunt levé par la société concessionnaire mais garanti par l'Etat et, si nécessaire, gagé sur un allongement des concessions existantes.

Ce dispositif vient d'être réformé pour cause d'incompatibilité avec la législation européenne et cette réforme va dans le sens d'une intervention plus importante de partenaires privés. Elle va aussi dans le sens d'une logique de financement mixte par le contribuable et l'usager-payeur.

\subsection{La logique mixte}

Le nouveau système mis en place pour les autoroutes concédées résulte, en effet, de la transposition de la directive communautaire 93-37 et abolit la discrimination dont bénéficiaient jusque là les sociétés d'autoroutes installées. Mais, du même coup, il abolit également ce mode de financement dit d'adossement, fort commode pour une puissance publique dispensée de toute contribution. C'est ainsi que les nouveaux appels à concession voient apparaître des opérateurs privés qui étaient auparavant hors jeu, mais aussi des subventions d'équilibre qui, jusqu'ici, n'étaient pas nécessaires car elles pouvaient être camouflées dans les recettes à venir de tronçons financièrement amortis.

Il appartient alors à chaque candidat d'établir son évaluation financière du projet sur la base de ses propres prévisions de coûts et de recettes, de négocier avec une banque ou un pool bancaire la part d'emprunt qui viendra compléter les fonds propres engagés et de proposer une offre qui précisera, en particulier, le montant de subvention nécessaire à l'équilibre de l'opération. Cet équilibre étant entendu avec la charge de la dette et la rémunération des fonds 
propres comprises, le montant de subvention proposé dépendra, d'une part, de la consistance technique et économique du dossier, d'autre part, de la situation des marchés financiers.

Le premier cas traité dans le nouveau dispositif (et ne résultant pas d'une annulation d'une concession accordée sous l'ancien) a été celui de la section Rouen - Alençon de l'autoroute A28 longue de $120 \mathrm{~km}$. Elle a été concédé à un groupement privé (Bouygues, EGIS et $\mathrm{CDC}$ ) et prévoyait une subvention d'équilibre d'environ $50 \%$ du coût de l'investissement.

Dans certains cas, il peut se trouver qu'un ou plusieurs candidats aboutissent à une rentabilité financière suffisante pour que le projet puisse être lancé sans subvention, comme cela fut possible pour le viaduc de Millau. Mais il s'agit généralement de situations très particulières tels le cas d'un court "maillon manquant» du réseau ou le cas de ce viaduc, desservi à ses deux extrémités par une autoroute libre de péage et, par conséquent, bien situé pour internaliser avec son péage du surplus des usagers de cette autoroute.

Il reste que, dans la majorité des cas, la puissance publique est sollicitée pour cofinancer le projet. Le candidat retenu a alors toutes les chances d'être celui qui demande la subvention la plus faible.

Les investissements ferroviaires des lignes à grande vitesse étaient initialement autofinancés, au même titre que les autoroutes à péage jusqu'à la fin des années 90 . Mais, si le TGV Sud-Est a largement couvert le service de la dette et son amortissement et si le TGV Atlantique a bénéficié d'une subvention qui ne lui était pas nécessaire, les rentabilités financières des liaisons qui ont suivi se sont révélées trop faibles pour épargner le contribuable.

La SNCF, puis, Réseau Ferré de France après la réforme de notre système ferroviaire en 1997 ont connu une situation comparable à celle d'un concessionnaire autoroutier dans le nouveau système. S'agissant des investissements soumis à l'article 4 du décret qui fonde RFF, c'est-à-dire des investissements de développement du réseau, il incombe, en effet, au gestionnaire d'infrastructure de n'investir que si les conditions de financement ne sont pas de nature à compromettre, dans la durée, son compte de résultat. Cela signifie que les produits apportés par l'investissement (péages et économies de dépenses d'entretien) doivent permettre de couvrir les coûts de fonctionnement futurs et la charge de l'emprunt levé pour cette opération. Plus précisément, le taux de rentabilité interne (TRI) de l'opération devra apporter cette garantie financière compte tenu des taux d'intérêt du marché et d'une prime de risque.

Comme pour la plupart des autoroutes concédées qui restent à construire, les liaisons nouvelles à grande vitesse et, plus généralement, les investissements ferroviaires ont des taux de rentabilité financière inférieurs au TRI qui doit être garanti à l'opérateur. Une subvention est donc également nécessaire pour que cette garantie soit effective. Cette contribution renvoie, comme dans le cas d'un pur financement public, à l'utilité collective qui la justifie. Une telle situation est, en somme, relative aux projets pour lesquels $\mathrm{VAN}_{\mathrm{f}}<0$ et $\mathrm{VAN}_{\mathrm{e}}>0$.

En nous inspirant du cas de la France, nous avons présenté cette évolution vers la logique mixte comme une dérive du cas de financement par le seul usager, dérive consécutive à des rentabilités financières décroissantes. Si l'on prend quelque recul pour considérer ce qui s'est passé dans le monde depuis les années 90, il est clair que la logique mixte résulte le plus souvent d'un amendement à la logique de l'utilité collective, qu'il s'agisse de pays éligibles aux prêts de la Banque Mondiale (Budin et Thompson, 2001) ou des pays de l'OCDE (Molnar, 2003). Le moteur de cette évolution, qui conduit au même résultat que la première, est alors la rareté des fonds publics.

Indépendamment des dispositifs de financement qui l'ont précédée, cette logique mixte pose un évident problème d'évaluation: dès lors que les financements de l'usager et du contribuable se combinent, quel est le bon indicateur pour désigner les priorités de réalisation ? Etant entendu que l'objectif demeure d'optimiser, sous une contrainte budgétaire active, la $\mathrm{VAN}_{\mathrm{e}}$ globale d'une séquence de projets qui constitue un programme, peut-on 
réduire le rôle du TRI à celui de condition permissive ? Nous allons montrer que la réponse est négative.

\section{Rentabilité financière et besoin de subvention}

Nous devons, dans cette deuxième section, établir une expression du besoin de subvention d'un projet en fonction de ses caractéristiques financières afin de pouvoir formaliser le rôle de la contrainte budgétaire. Cette formalisation a déjà été proposée pour une tout autre fin (Bonnafous, 2002) : il s'agissait, en effet de comparer l'intérêt pour les finances publiques d'un recours à un opérateur public ou à un opérateur privé, le premier ayant l'avantage de ne pas exiger une rémunération des fonds propres, le second celui de pouvoir gagner quelques points sur la rentabilité financière du projet s'il se montre plus efficace que l'opérateur public.

Nous reprenons, par conséquent, cette formalisation sans laquelle nous ne pourrions expliciter le problème de l'optimisation sous contrainte budgétaire du choix des investissements. Il s'agit d'exprimer le besoin de subvention requis pour atteindre le TRI qui doit être garanti à l'opérateur.

\subsection{Formalisation du besoin de subvention}

Pour cela, considérons un projet qui correspond à une chronique stylisée, mais somme toute classique, des coûts et des bénéfices représentée sur la figure 1. Si la mise en service est supposée réalisée à la date $t=0$, la dépense annuelle entre les dates $-d$ et 0 est de $c$. A partir de la mise en service, le bénéfice dégagé est supposé de la forme $(a+b . t)^{14}$.

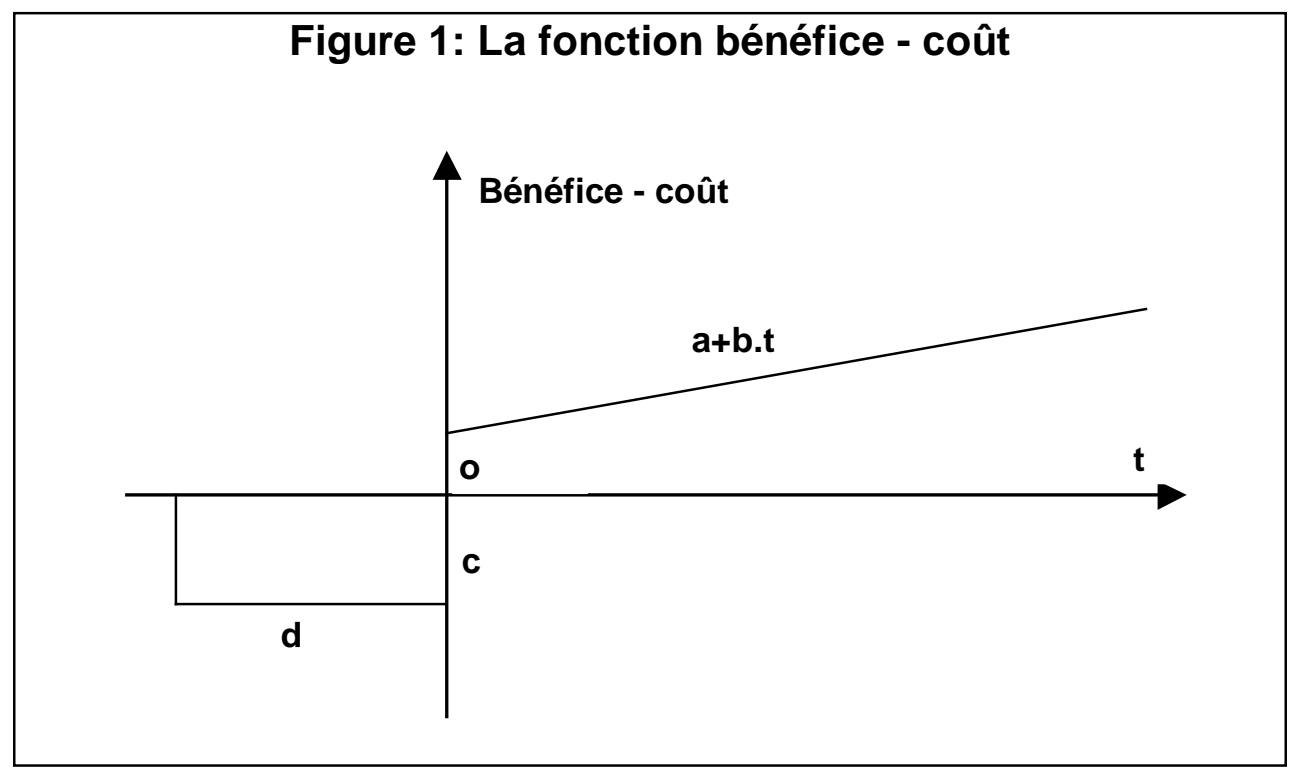

Le taux de rentabilité interne du projet (TRI), c'est à dire le taux d'actualisation qui annule sa valeur actualisée nette $V A N_{f}$, est alors une fonction des quatre paramètres $c, d, a$ et $b$. Il est à comparer au taux de rentabilité qu'un opérateur (public ou privé) est en droit d'escompter.

Nous utiliserons les notations suivantes :

- $\quad \alpha$ est le taux d'actualisation utilisé pour calculer la $V A N_{f}$, 
- $\quad \alpha_{0}$ est le taux d'actualisation qui annule la $V A N_{f}$ du projet, c'est à dire son TRI,

- $\delta$ est le supplément de TRI que la subvention apporte à l'opérateur,

- $\tau$ est le taux de subvention de l'investissement, soit la part de $c$ financée par subvention.

Pour un taux d'actualisation $\alpha$, et un bilan actualisé calculé des dates $-d$ à $T$, la valeur actualisée nette du projet s'écrit :

$$
V A N_{f}=\int_{-d}^{0}-c \cdot e^{-\alpha . t} \cdot d t+\int_{0}^{T}(a+b \cdot t) \cdot e^{-\alpha . t} \cdot d t
$$

Nous supposerons que l'actualisation est étendue à l'infini, ce qui est sans conséquence sur les résultats qui nous intéressent en raison du faible poids du futur lointain et, surtout, de la convergence des fonctions intégrales de l'équation (1). Cette équation devient alors ${ }^{15}$ :

$$
V A N_{f}=\frac{1}{\alpha}\left[c\left(1-e^{\alpha . d}\right)+a+\frac{b}{\alpha}\right]
$$

Le TRI du projet, $\alpha_{0}$, est alors donné par :

$$
c\left(1-e^{\alpha_{0} \cdot d}\right)+a+\frac{b}{\alpha_{0}}=0
$$

Un taux de subvention $\tau$ abaisse le coût annuel de construction $c$ à $c(1-\tau)$ et porte le TRI $\alpha_{0}$ à $\left(\alpha_{0}+\delta\right)$ de sorte l'équation (3) devient :

$$
(1-\tau) c\left(1-e^{\left(\alpha_{0}+\delta\right) d}\right)+a+\frac{b}{\alpha_{0}+\delta}=0
$$

Dont nous pouvons déduire l'expression du taux de subvention :

$$
\tau=1-\frac{a\left(\alpha_{0}+\delta\right)+b}{c\left(\alpha_{0}+\delta\right)\left(e^{\left(\alpha_{0}+\delta\right) d}-1\right)}
$$

La relation entre $\tau$, le taux de subvention, et $\delta$, l'augmentation du TRI du projet qu'il s'agit d'apporter à l'opérateur, dépend, évidemment, des paramètres $c, d, a, b$ et, bien sûr, $\alpha_{0}$, qui caractérisent la rentabilité financière initiale du projet. Ces paramètres sont, en outre, liés entre eux par l'équation (3) qui définit le TRI du projet $\alpha_{0}$ (ou ce qui est équivalent, $\tau=0$ si et seulement si $\delta=0$ ). Cela implique quelques difficultés dans l'étude de la fonction dont il nous faut pourtant exprimer les propriétés.

\subsection{Propriétés de la fonction de subvention}

Si nous voulons représenter l'équation (5), il nous faut figer certains de ces cinq paramètres et ne faire varier que ceux dont nous souhaitons exhiber le rôle. Nous aurons recours, pour cela, à la technique classique des abaques. Nous ne représenterons qu'un seul de ces abaques (figure 2), qui sera suffisant pour illustrer notre propos. Le coût annuel de construction $c$ a été fixé à une valeur normée de 100, la durée $d$ de cette construction est fixée à 5 ans. L'accroissement annuel des avantages nets des coûts, $b$, est supposé égal à 1 . Cela 
revient à faire varier le TRI initial du projet $\alpha_{0}$ (ou encore $a$, puisque $\alpha_{0}$ ne dépend plus que de $a$, bénéfice net du projet à la date de mise en service).

L'abaque ci-dessous présente ainsi la fonction (5) sous contrainte de l'équation (3) pour une série de valeurs de $\alpha_{0}$, entre $2 \%$ et $14 \%$ avec un pas de $0.4 \%$ (ce qui correspond à des valeurs croissantes de a de gauche à droite sur le graphique). Pour chacune de ces valeurs de $\alpha_{0}$, qui se lit en abscisse, chaque courbe exprime le taux de subvention nécessaire pour élever le TRI au niveau $\left(\alpha_{0}+\delta\right)$.

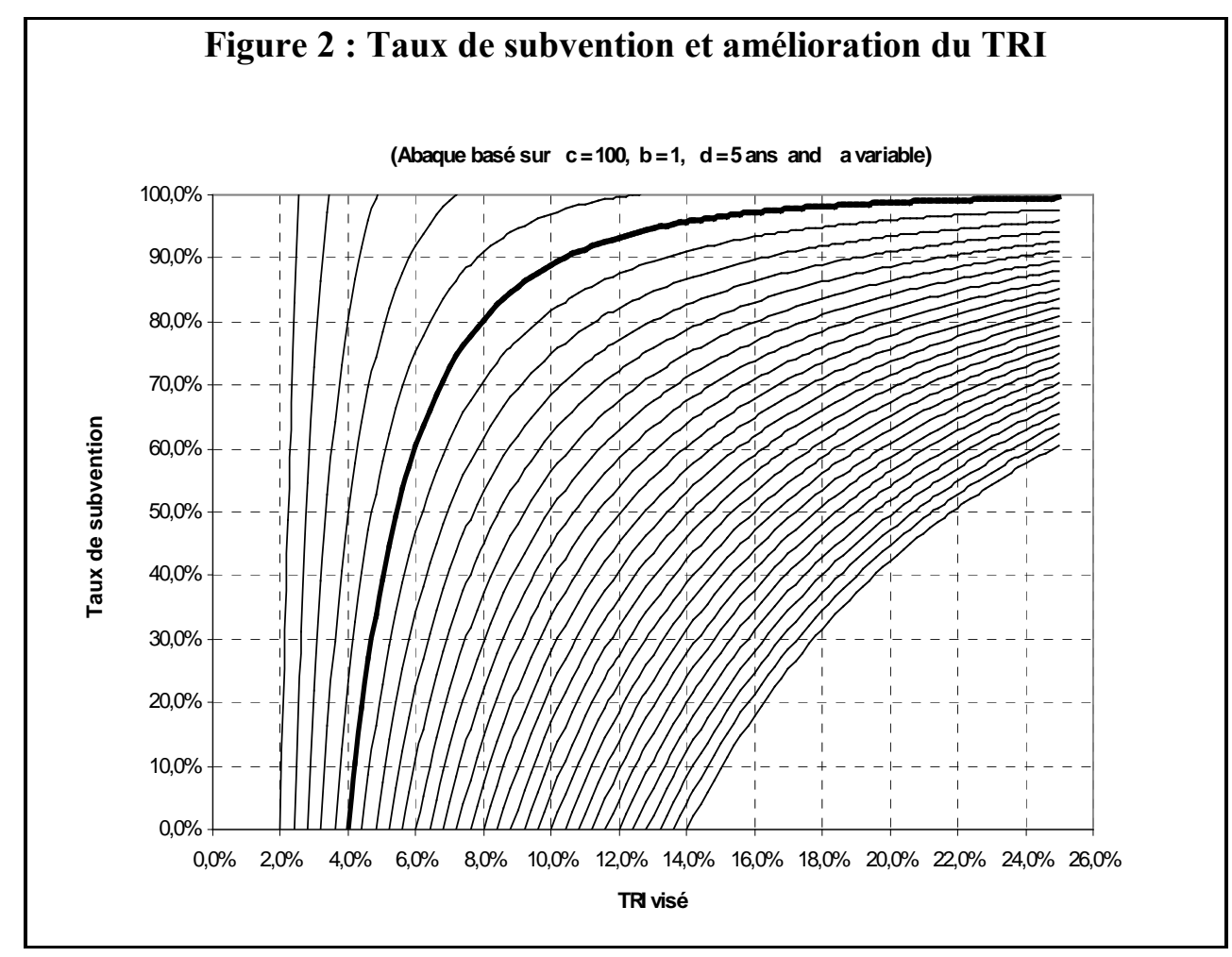

Si elle reposent sur les caractéristiques de la série chronologique des coûts et avantages du projet considéré et sur les valeurs particulières retenues pour certains paramètres, les silhouettes de ces courbes ont un caractère général. En particulier, leur concavité résulte des propriétés de la dérivée seconde de la fonction (5) qui peuvent être démontrées sous la condition que b soit positif. De cette concavité résultent des conséquences importantes quant à l'alternative entre un opérateur public et un partenariat privé qui ont été précisées par ailleurs (Bonnafous, 2002). Mais la première de ces conséquences concerne le rôle croissant de la rentabilité financière intrinsèque $\alpha_{0}$ à mesure que celle-ci s'affaiblit.

On pouvait s'attendre à ce que le besoin de subvention soit une fonction croissante du taux de rentabilité interne qu'il s'agit d'assurer à l'opérateur, mais sa concavité constitue un résultat plus inattendu : cela signifie, en particulier, que les premiers écarts entre le TRI initial de l'opération et le TRI visé sont très coûteux alors que l'on s'attend, intuitivement, à ce qu'une injection de subvention dans un projet fasse croître rapidement la rentabilité pour l'opérateur.

Cela signifie que l'effet de levier des finances publiques sur le rythme de réalisation des investissements est beaucoup plus sensible qu'on ne le soupçonne généralement au fait que l'on accorde ou non une priorité aux projets qui ont la meilleure rentabilité financière. 


\section{Conséquences sur la détermination du programme optimal}

Le besoin de financement public étant d'autant plus grand que le taux initial de rentabilité financière est faible et étant rapidement croissant dès lors qu'il s'agit de la relever de quelques points, les capacités de financement public peuvent être beaucoup plus vite épuisées si, au motif qu'ils ont une bonne rentabilité socio-économique, on réalise en priorité des projets de faible rentabilité financière.

\subsection{La tyrannie de la rentabilité financière}

Ainsi, alors que le TRE est sensé désigner les projets qui apportent le plus fort rendement social, il n'est plus dit qu'en réalisant les investissements dans l'ordre (décroissant) de leur rentabilité socio-économique on obtienne un meilleur rendement social global que lorsque l'on favorise les projets à fort rendement financier. Dans ce cas, en effet, les rares ressources publiques permettront de réaliser plus de projets et cela peut produire, au total, plus de surplus socio-économique que l'ordre de réalisation suggéré par les TRE.

Des simulations de programmes d'investissement ont été ainsi réalisées pour donner quelque consistance à cette conjecture ${ }^{16}$. Ces simulations, comme celles de la sous-section 3.2 reposent sur une simplification très abusive: les caractéristiques socio-économiques et financières des projets sont supposées indépendantes de la date de mise en service. La nécessaire dynamisation de l'exercice sera évoquée in fine.

L'exercice a consisté à traiter un ensemble de 17 projets d'autoroutes à péage pour lesquels les éléments nécessaires à ces simulations étaient connus et évalués selon des méthodes homogènes ${ }^{17}$ ou ont pu être reconstitués à l'aide du modèle présenté dans la soussection 2.1. Ainsi, les taux de subvention ont été calculés sur la base des équations (3) et (5), qu'elles soient appliquées au bilan actualisé socio-économique ou au bilan financier. Il a été supposé, dans un premier temps, que les projets étaient réalisés sous une contrainte budgétaire limitant le financement public annuel à 150 millions d'euros lors de la première année du programme, ce financement étant supposé augmenter ensuite de 2,5\% par an.

Quatre simulations sont proposées qui supposent, respectivement, que les projets sont réalisés dans l'ordre d'un TRI décroisant, dans l'ordre du TRE, puis deux ordres aléatoires (correspondant à l'ordre alphabétique et à son inverse). Chacun de ces programmes, qui est supposé durer 15 ans, a évidemment un certain rendement social que nous choisissons de synthétiser par le ratio entre la $\mathrm{VAN}_{\mathrm{e}}$ (ou surplus collectif) que dégage le programme rapportée à l'euro de subvention. Les résultats sont reportés dans le tableau ci-dessous, complétés par une information relative à la longueur du réseau créée par chaque programme sur laquelle nous reviendrons.

Tableau 1 : Rendement social d'un programme de 17 projets d'autoroutes à péage selon l'ordre de réalisation des projets

\begin{tabular}{lcccc}
\hline $\begin{array}{l}\text { Ordre de réalisation des } \\
\text { projets }\end{array}$ & $\begin{array}{c}\text { Ordre des } \\
\text { TRI } \\
\text { décroissants }\end{array}$ & $\begin{array}{c}\text { Ordre des } \\
\text { TRE } \\
\text { décroissants }\end{array}$ & $\begin{array}{c}\text { Ordre } \\
\text { alphabétique }\end{array}$ & $\begin{array}{c}\text { Ordre } \\
\text { alphabétique } \\
\text { inversé }\end{array}$ \\
\hline $\boldsymbol{V A N}_{\boldsymbol{e}} /$ euro public obtenue & 4,27 & 3,26 & 2,19 & 0,77 \\
Longueur du réseau réalisé & 525 & 485 & 335 & 330 \\
\hline
\end{tabular}

Source : séminaire de recherche du LET (Brunel, 2003)

Relevons tout d'abord que ce tableau souligne les mauvais résultats, au sens de l'efficacité économique, que pourrait engendrer la réalisation d'un programme selon un ordre 
aléatoire, ici alphabétique ou alphabétique inverse, mais pouvant tout aussi bien être celui qui résulterait de l'influence politique de notables locaux.

Ces résultats nous livrent une leçon plus importante encore, et surtout moins connue, car ils remettent en cause un principe généralement admis en économie publique selon lequel les TRE des projets désignent ceux qu'il convient de réaliser en priorité pour dégager le meilleur rendement social. Or, dans cet exemple, le surplus collectif du programme rapporté à la subvention ( $\mathrm{VAN}_{\mathrm{e}} /$ euro public) est meilleur lorsque l'on choisit l'ordre des TRI plutôt que celui des TRE.

Il s'agit évidemment d'un effet de la contrainte financière : lorsque la capacité de financement public est faible, un programme d'investissement qui ne tient pas compte du TRI épuise très vite le budget public disponible et cela entraîne un rythme de mise en service ralenti. La dernière ligne du tableau 1 exprime clairement cet « effet budgétaire » et explique le paradoxe par la longueur de réseau mis en service selon le programme réalisé : sous contrainte budgétaire, la négligence pour la rentabilité financière conduit à sacrifier quelques nouveaux kilomètres de réseau et à renoncer à la part de $\mathrm{VAN}_{\mathrm{e}}$ qu'ils induisent.

On peut supposer que cette conjecture est d'autant plus probable que les capacités de financement sont faibles. A la limite, avec des capacités inépuisables de financement public, tous les projets pourraient être réalisés au plus tôt. Pour illustrer cet effet de la contrainte budgétaire, les simulations de programme ont été diversifiées en desserrant la contrainte budgétaire (de 150 à $600 \mathrm{M} €$ ). Les résultats sont présentés sur la figure 3 ci-dessous.

On voit bien ici que l'effet budgétaire s'estompe à mesure que la contrainte de financement public se desserre. Lorsque celui-ci atteint 600 millions d'euros, la totalité des projets considérés (soit $1105 \mathrm{~km}$ d'autoroutes nouvelles) peut être réalisée dans la durée du programme. En ce cas, l'explication du paradoxe en termes de longueur de mises en service sur la durée du programme ne joue plus, mais il reste que les ordres de réalisation aléatoires produisent moins de surplus global car les projets à forte rentabilité et qui dégagent les surplus les plus importants n'ont pas été mis en service les premiers.

Relevons au passage que cet éclairage du rôle variable de la contrainte budgétaire nous suggère qu'il est somme toute logique que la prise en compte de considérations d'aménagement du territoire ne survienne, historiquement, qu'à partir du moment où un certain niveau d'aisance des finances publiques est atteint : non seulement c'est le signe que les investissements les plus urgents ont été réalisés mais, de surcroît, c'est aussi une situation dans laquelle nos résultats montrent que la perte sociale est limitée lorsque le choix est fait de privilégier des investissements de faible rentabilité. 


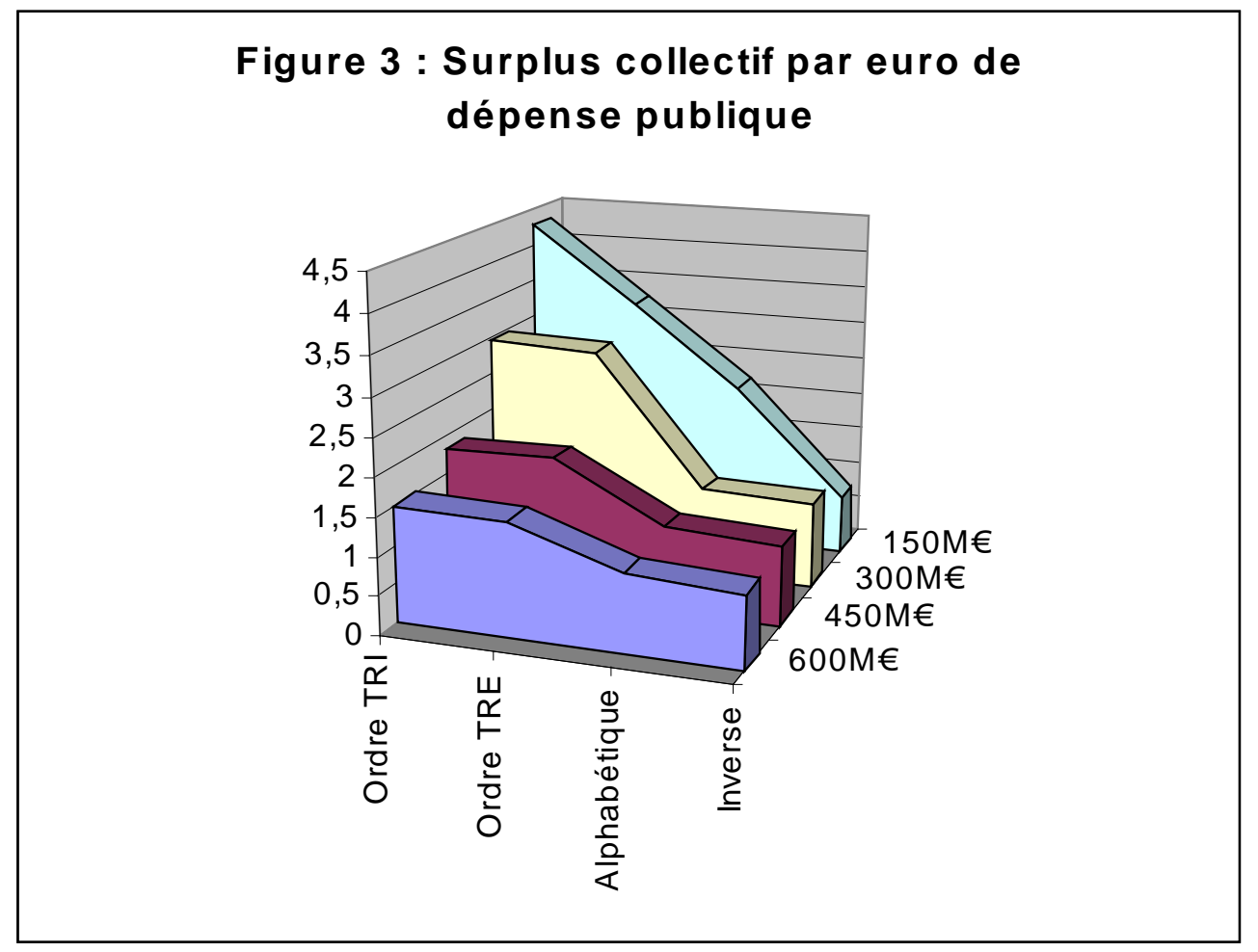

Retenons, enfin, que ces simulations, qui suggèrent d'accepter la tyrannie de la rentabilité financière lorsque les ressources publiques sont limitées, dépendent évidemment de la configuration des projets qui constituent les programmes alternatifs. Il peut y avoir pour chacun des programmes des « effets de réseau » tels, par exemple, que l'ordre de réalisation des projets ait des conséquences sur la rentabilité de chacun. Nous ne proposons donc ici qu'une conjecture qui peut s'exprimer ainsi : un programme de réalisation des investissements dans l'ordre (décroissant) de leur rentabilité socio-économique peut avoir un moins bon rendement social global qu'un programme qui privilégie les projets à fort rendement financier. Cela est d'autant plus probable que les capacités de financement public sont limitées. Mais la question du programme optimal reste entière.

\subsection{La détermination du programme optimal}

Dans la mesure où il n'y a pas de relation fonctionnelle entre le TRI et le TRE d'un projet (voir fig. 5 en annexe), il n'y a pas de raison particulière pour que l'ordre des TRI dégage le programme optimal, au sens où, sous la même contrainte budgétaire, il n'y en aurait aucun autre qui dégagerait un surplus global plus élevé. On peut même avancer, au moins de manière intuitive, qu'un ordre de programmation fondé sur les valeurs décroissantes du ratio $\mathrm{VAN}_{\mathrm{e}}$ par euro de subvention doit produire la meilleure $\mathrm{VAN}_{\mathrm{e}}$ globale possible.

Comme quelques uns des résultats qui précèdent suggèrent qu'il faut se garder des intuitions, il convient de tenter d'apporter une démonstration à ce principe d'optimisation. Mais nous sommes là face à un problème qui ne se soumet guère à une démonstration analytique $^{18}$ dès lors qu'il s'agit de prendre en compte la diversité des projets, le caractère discret du problème et le nombre considérable de solutions. Ainsi, la série des projets considérés au précédent paragraphe peut donner lieu à 17 ! permutations possibles (environ $10^{34}$ ). Les algorithmes d'exploration combinatoire semblent apporter quelques résultats dans les sciences expérimentales qui sont confrontées à ce genre de problème, par exemple dans les modèles évolutionnaires de la biologie ou dans l'exploration des nanostructures. C'est à l'une 
de ces méthodes que nous avons eu recours. Elle a été expérimentée (Bonnafous et Jensen, $2004^{19}$ ) en retenant le même paquet des 17 projets utilisé précédemment.

La fonction objectif reste, bien entendu, la $\mathrm{VAN}_{\mathrm{e}}$ globale du programme de projets réalisé sous contrainte de financement public. L'algorithme est issu de la méthode des recuits simulés (van Laarhoven, 1992) et consiste, en partant d'un ordre aléatoire des projets, à simuler des permutations entre deux projets, eux-mêmes étant désignés par un processus aléatoire. Toute permutation qui augmente la fonction objectif, ou qui ne la diminue que d'un faible niveau (niveau qui décroît au cours de la simulation, pour assurer la convergence), est conservée. La contrainte de financement public F est introduite de la manière suivante. Pour chaque projet individuel, nous calculons d'abord une durée de réalisation en tenant compte de la valeur de F. Cette durée, calculée en utilisant l'équation (5), rend son financement possible dans ce laps de temps, c'est-à-dire que le montant annuel de subvention nécessaire à la réalisation du projet est alors au plus égal à $\mathrm{F}$. Nous pouvons alors entamer la simulation du programme pour cette valeur de F. Le programme «prend» le premier projet. S'il reste un reliquat de subvention (cas où la subvention nécessaire à ce premier projet serait inférieure à $\mathrm{F}$ ), il est affecté au démarrage du deuxième projet. A la fin de la première année, le premier projet avance d'une année, et le deuxième d'un pourcentage d'année, égal à la proportion de sa subvention annuelle couverte par le reliquat laissé par le premier projet. L'algorithme est évidemment repris pour différentes valeurs de $\mathrm{F}$ que nous faisons varier autour d'une valeur centrale $(<\mathrm{F}>)$.

La fonction objectif se stabilise après quelques milliers d'itérations et toutes les expériences, menées avec des ordre initiaux et des paramètres variables, voient la fonction objectif converger vers la même valeur.

Nous ne retiendrons ici que le principal résultat de cet exercice qui est figuré sur la figure 4 ci-après. Il représente, en abscisse, la contrainte financière rapportée à une valeur moyenne et, en ordonnée, le surcroît de valeur actualisée nette par rapport au programme ordonné selon les TRE des projets.

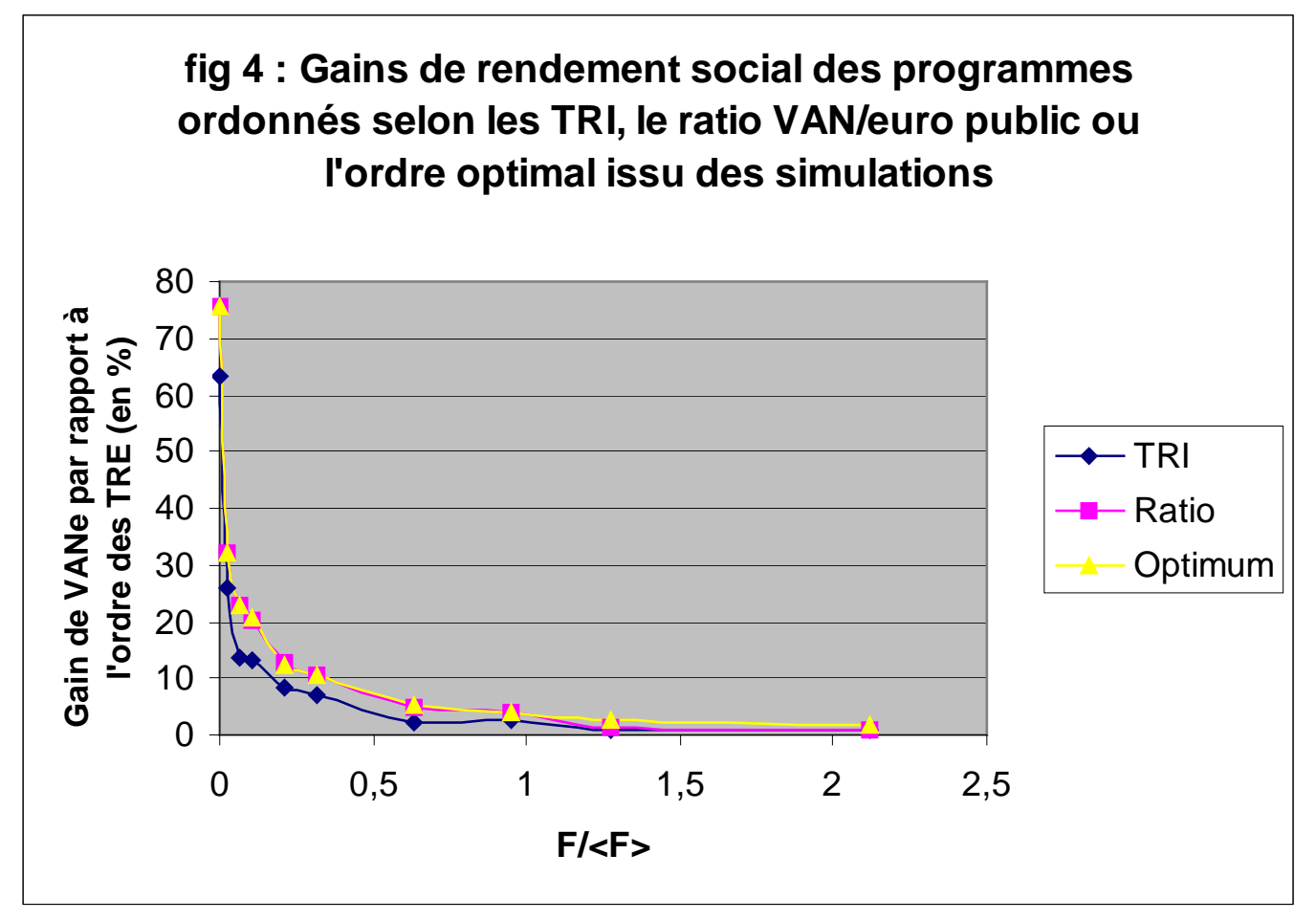

Ces résultats confirment que ce gain de rendement social est nettement positif avec l'ordre du TRI et cela d'autant que la contrainte budgétaire est resserrée. Mais ils nous 
confirment surtout (nous démontrent au sens des physiciens) que l'ordre du ratio $\mathrm{VAN}_{\mathrm{e}}$ / euro public investi apporte un gain de rendement social supérieur encore et que c'est bien le critère qui désigne le programme optimal.

La figure 4 nous suggère également que lorsque la contrainte budgétaire est desserrée, il n'y a pas grand chose à gagner, pour la collectivité, par rapport à l'ordre des TRE décroissants. Cet ordre est celui qui a largement inspiré les choix de priorité pendant des décennies même si, parfois, le taux de rentabilité immédiate (socio-économique) dont le calcul est plus aisé que celui du TRE a pu jouer ce rôle. Le graphique ci-dessus nous suggère que la perte de rendement social par rapport à un ordre plus profitable pour la collectivité n'a pas été considérable. En revanche, il nous suggère aussi que cette perte prend de l'ampleur à mesure que la contrainte budgétaire devient plus forte. Il nous indique, en particulier, que dans un contexte de grande rareté des fonds public qui est celui des pays en développement il peut y avoir un avantage très sensible à raisonner en termes de rentabilité financière ou, mieux encore, du ratio proposé.

Il va de soi que notre exercice repose sur quelques hypothèses simplificatrices dont nous ne sommes pas assurés qu'elles résisteraient à l'incertitude liée à la longue durée ou encore à des dynamiques très singulières de coûts et avantages. Les projets qui sont mis en concurrence par la contrainte budgétaire ne sont, bien entendu que des projets « réellement en attente », ce qui veut dire, au sens du calcul économique, qu'ils vérifient deux conditions : d'une part, leur VAN socio-économique ne doit pas être négative et, d'autre part, leur rentabilité immédiate doit être au moins égale au taux d'actualisation (Abraham et Laure, 1959). A l'exception d'un seul, les projets de la base de données utilisée ci-dessus remplissent les deux conditions. Ainsi, le critère de hiérarchisation des projets que nous proposons a pour objet de déterminer l'ordre de réalisation des projets qui maximise le rendement social sous une contrainte budgétaire, mais n'a pas vocation à se substituer à celui d'Abraham et Laure, qui a pour objet de déterminer la date optimale de mise en service sans contrainte budgétaire ${ }^{20}$. Or cette contrainte a généralement pour effet de différer les projets par rapport à leur date optimale de réalisation et, ainsi, d'impliquer la vérification des deux conditions ci-dessus.

\section{Le programme optimal : modélisation}

Nous utilisons une modélisation proche de celle de Weingartner (1963), lorsqu'il se proposa de résoudre le problème fondamental posé par Lorie \& Savage $(1955)^{21}$. Toutefois, ces auteurs se sont concentrés sur une résolution par programmation linéaire, qui ne permet pas de produire un résultat général.

Nous supposerons dans ce qui suit que le décideur souhaite choisir parmi $n$ projets $i$, caractérisés par leur valeur actualisée nette ${ }^{22} V A N_{i}$ et leur besoin de subvention $S_{i}$, avec $V A N_{i}$ et $S_{i}>0 \quad \forall i=1, \ldots, n$.

Le besoin de subvention est un montant qui représente tout ou partie des frais d'investissements (voire d'exploitation) nécessaires pour que l'État puisse amener une entreprise à s'engager dans un contrat de concession de type BOT (Built Operate and Transfert) sur un segment d'autoroute.

Nous retenons comme fonction objectif de la collectivité le surplus $W$ dégagé par l'ensemble des projets, sous contrainte du budget $B$ plafonnant les dépenses publiques d'investissement : 


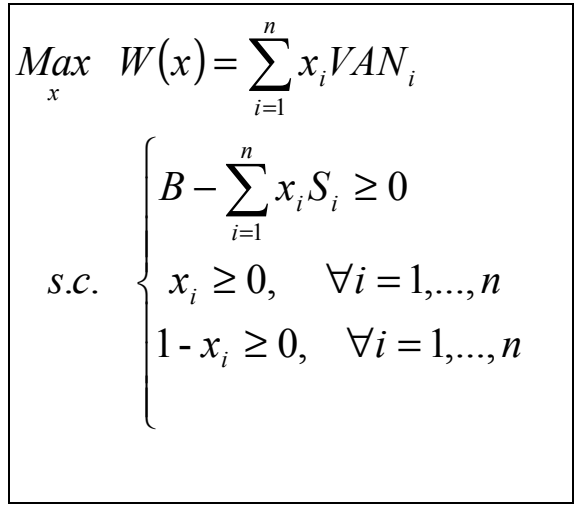

Les $x_{i}$ sont des variables continues, qui ont une valeur nulle lorsque le projet n'est pas réalisé et égale à l'unité lorsque le projet est réalisé en totalité. Nous supposerons qu'il est possible de réaliser partiellement un projet, le $x_{i}$ correspondant étant alors compris entre 0 et 1. Nous verrons que cette hypothèse de réalisation partielle des projets, tout à fait théorique, n'est pas très contraignante.

Cette modélisation possède l'avantage d'être relativement simple et de nature à faciliter l'interprétation des résultats. Elle contient une hypothèse décisive (concernant la réalisation partielle des projets) qui nous autorise à écrire un lagrangien sans postuler une relation fonctionnelle entre les paramètres $V A N$ et $S$. Les $2 n$ contraintes sur les $x_{i}$ permettent de transformer le problème de choix discret, pour le résoudre de manière analytique.

En pratique, les projets sont soit réalisés en totalité, soit ne le sont pas du tout. La possibilité de réaliser un projet partiellement, tout en bénéficiant proportionnellement de ses caractéristiques (VAN et $S$ ), est essentiellement un outil théorique. Cela dit, la réalisation partielle d'un projet ne sera in fine que marginale dans la solution du programme. Un projet au plus sera dans ce cas. Le vecteur solution $x^{*}$ est constitué d'un ensemble de 1 (projets réalisés), d'un ensemble de 0 (projets non réalisés), et d'une valeur comprise entre 0 et 1 pour le projet «limite» $x_{k}$. En supposant que les projets sont ordonnés selon leur priorité de réalisation, on a :

$$
x^{*}=(\underbrace{1, \ldots \ldots \ldots 1}_{\text {projets acceptés }}, x_{k}, \underbrace{0, \ldots \ldots \ldots, 0}_{\text {projets rejetés }})
$$

Ainsi, le meilleur projet sera réalisé en totalité, puis le deuxième, le troisième etc...jusqu'à saturation de la contrainte budgétaire. C'est le projet « limite » qui risque de ne pas être terminé, et seulement celui-là.

La figure 6 propose une application du programme d'optimisation avec deux projets. La VAN du projet 2 est supérieure à celle du projet 1 , et le niveau d'investissement identique $\left(I_{1}\right.$ $=I_{2}=25$ ). Pourtant c'est la réalisation du projet 1 en totalité et de $1 / 7^{e}$ du projet 2 qui permet d'atteindre la courbe d'indifférence la plus élevée. Ce résultat ne peut être compris qu'à la lumière des besoins de subvention, qui doublent quasiment d'un projet à l'autre. On peut remarquer que sur cet exemple, les projets sont rangés dans l'ordre du ratio $V A N / S$. 
Figure 6: Ordre optimal de réalisation sous contrainte financière, le cas de deux projets

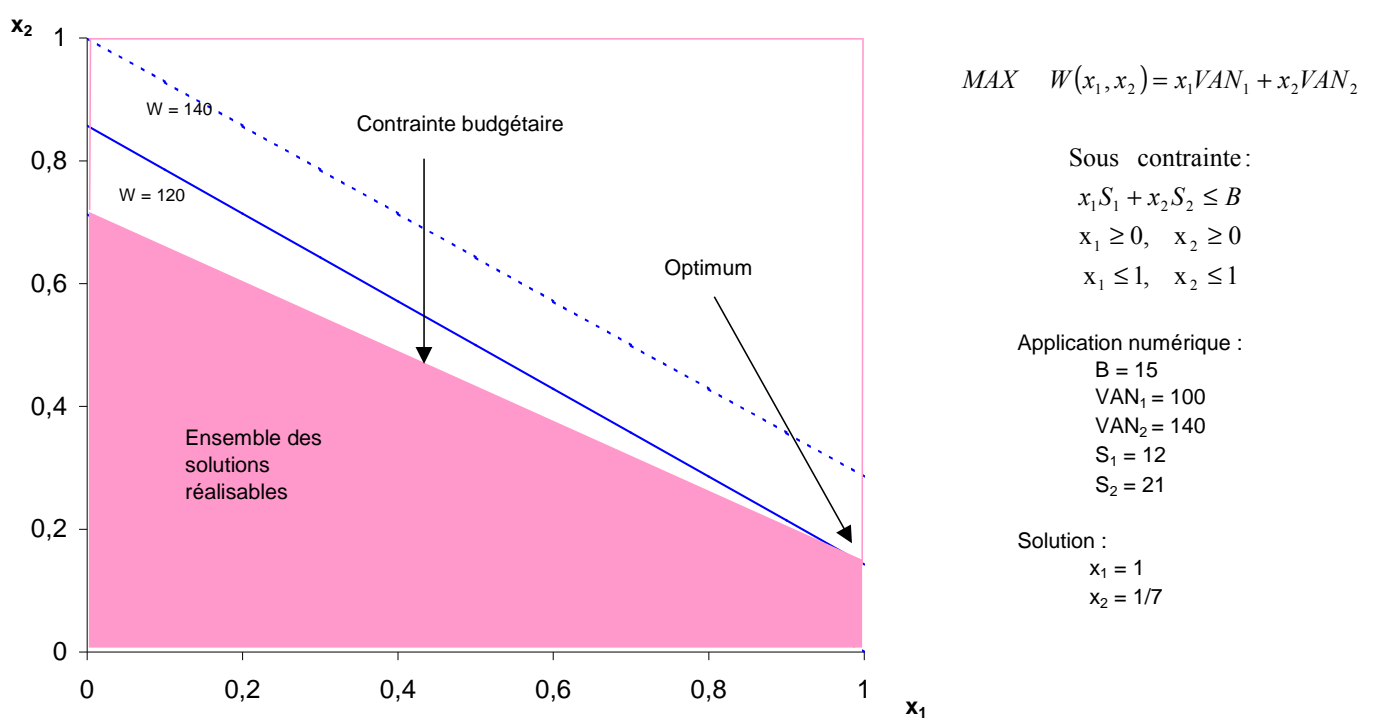

Le Lagrangien généralisé, incluant les contraintes de non-négativité des $x_{i}$, s'écrit :

$L\left(x_{1}, \ldots, x_{n}, \lambda, \alpha_{1}, \ldots, \alpha_{n}, \beta_{1}, \ldots, \beta_{n}\right)=\sum_{i=1}^{n} x_{i} V A N_{i}+\lambda\left(B-\sum_{i=1}^{n} x_{i} S_{i}\right)+\sum_{i=1}^{n} \alpha_{i} x_{i}+\sum_{i=1}^{n} \beta_{i}\left(1-x_{i}\right)$

Les conditions de Kuhn et Tucker impliquent notamment :

(6)

$V A N_{i}-\lambda S_{i}+\alpha_{i}-\beta_{i}=0, \forall \mathrm{i}=1, \ldots, \mathrm{n}$

(7) $\lambda\left(B-\sum_{i=1}^{n} x_{i} S_{i}\right)=0$

(8) $\quad \alpha_{i} x_{i}=0, \forall \mathrm{i}=1, \ldots, \mathrm{n}$

(9) $\beta_{i}\left(1-x_{i}\right)=0, \forall \mathrm{i}=1, \ldots, \mathrm{n}$

Les multiplicateurs de Kuhn et Tucker $\left(\lambda, \alpha_{i}\right.$ et $\left.\beta_{i}\right)$ sont non négatifs par définition. Ils sont positifs (ou exceptionnellement nuls) lorsque la contrainte qui leur est associée est saturée et nuls lorsque que cette contrainte est non saturée. Pour le projet « limite » $k$, vérifiant $0<x_{k}<1$, on a d'après (6) :

$$
V A N_{k}-\lambda S_{k}=0 \Leftrightarrow \frac{V A N_{k}}{S_{k}}=\lambda
$$

Dans le cas d'un desserrement marginal de la contrainte budgétaire à l'optimum, c'est uniquement la réalisation du projet $k$ qui va être affectée. On peut donc écrire les différentiels suivants à l'optimum :

$$
\begin{gathered}
d B=S_{k} d x_{k} \\
d W=V A N_{k} d x_{k}
\end{gathered}
$$

En combinant les équations (10), (11) et (12) on obtient : $\frac{d W}{d B}=\lambda$ 
$\lambda$ est dans cette formulation la variation de surplus collectif induite par un desserrement de la contrainte de disponibilité des fonds publics. C'est le montant maximum de surplus que la collectivité peut espérer obtenir avec une unité budgétaire supplémentaire.

Le multiplicateur $\lambda$ est associé à la contrainte financière de la collectivité. Plus la contrainte est forte, et plus $\lambda$ est élevé. A la manière d'un prix, dual de la contrainte, il représente le coût d'opportunité des fonds publics ${ }^{23}$. C'est un indicateur de rareté des ressources budgétaires.

Pour les projets acceptés (indicés $j$ ), les conditions de Kuhn et Tucker impliquent :

- Que la contrainte $« x_{i} \geq 0 »$ n'est pas saturée, on a donc $\alpha_{j}=0$

- Que la contrainte $« 1-x_{i} \geq 0 »$ est saturée, on a donc $\beta_{j}>0$

D'où $V A N_{j}-\lambda S_{j}>0 \Leftrightarrow \frac{V A N_{j}}{S_{j}}>\lambda$

L'ensemble des projets acceptables n'est pas composé de l'ensemble de ceux disposant d'une VAN positive. Ne sont retenus que ceux ayant un ratio $V A N / S$ supérieur au coût d'opportunité de l'argent public $\lambda$.

Pour les projets $l$ rejetés ou différés, les conditions d'optimisation impliquent :

- Que la contrainte « $x_{i} \geq 0 »$ est saturée, on a donc $\alpha_{l}>0$

- Que la contrainte $« 1-x_{i} \geq 0 »$ n'est pas saturée, on a donc $\beta_{l}=0$

D'où $V A N_{l}-\lambda S_{l}>0 \Leftrightarrow \frac{V A N_{l}}{S_{l}}<\lambda$

Les projets rejetés ont un ratio $V A N / S$ inférieur au coût d'opportunité des fonds publics.

Au total, (13) et (14) impliquent: $\frac{V A N_{j}}{S_{j}}>\lambda>\frac{V A N_{l}}{S_{l}}$

Les projets acceptés doivent toujours avoir un ratio $V A N / S$ supérieur à celui des projets rejetés. On privilégie ainsi les projets produisant le plus de valeur par euro public investi.

Si on fait varier $\lambda$ (desserrement ou resserrement de la contrainte budgétaire) on construit une hiérarchisation totale des priorités selon le critère $V A N / S$. Pour optimiser le surplus global sous contrainte budgétaire, lorsque le décideur ignore $\lambda$, il doit choisir en priorité les projets disposant d'un ratio « utilité collective par euro public investi » le plus élevé.

Le modèle que nous avons développé, qui aboutit au même résultat lorsque l'on introduit une VAN fonction du temps (Roy 2005), montre que le critère "VAN par euro public investi » identifié dans la section précédente a des bases théoriques relativement solides pour soutenir cette hiérarchisation.

Soulignons que ce critère est tout à fait conforme aux résultats standards de la microéconomie. Sa signification a un lien direct avec la théorie du consommateur : à l'équilibre de son optimisation d'utilité, le consommateur égalise tous les rapports entre utilité marginale et prix. Notre modélisation permet simplement de relâcher les hypothèses de divisibilité des quantités consommées et d'utilité variable selon la quantité consommée. Le résultat n'en est que plus acceptable pour traiter le cas d'investissements indivisibles et uniques par nature. Le consommateur considéré est la collectivité dans son rôle de fourniture de biens (en partie) non excludables. Et l'avancée consiste à montrer qu'il ne faut pas considérer l'investissement global, mais uniquement la dépense consentie directement par la collectivité. 
La pratique avait plutôt consacré le ratio entre les avantages bruts actualisés $(V A)$ et le coût d'investissement $I$, appelé ratio bénéfices-coûts ${ }^{24}$. Il correspond au ratio $V A N / I$ à une transformation croissante près. On comprend aisémment qu'il ait été utilisé utilisé dans des pays $^{2}$ qui ne pratiquaient pas le péage routier, comme au Royaume-Uni ${ }^{25}$ et en Allemagne ${ }^{26}$ ou au Japon qui le pratiquait mais avec des entreprises publiques ${ }^{27}$ (Hayashi \& Morisugi 2000, table 9). C'est aussi le cas dans les entreprises, où l'on utilise ce qui est appelé "l'indice de profitabilité ${ }^{28}$, qui considèrent bien entendu au numérateur la $\mathrm{VAN}_{\mathrm{f}}$ et non la $V_{A N}$. En somme le VAN/I se justifie dès lors que le I fait l'objet d'un financement homogène dans lequel le contribuable finance tout ou ne finance rien.

En France, sous la pression du financement mixte, la doctrine s'est récemment affinée pour prendre acte : dans une lettre le 27 mai 2005 portant mise à jour de l'instruction - cadre du 25 mars 2004, le ministère français des transport a redéfini les modalités de classement des grands projets d'infrastructure de transport : «afin de tirer le meilleur parti d'un financement public limité, la règle de classement des projets ${ }^{29}$ doit être non pas le bénéfice actualisé induit par le projet, mais le bénéfice actualisé par euro public dépensé » (Annexe III-§4).

L'innovation est donc au dénominateur: on n'y trouve pas le montant investi, mais «l'euro public dépensé ». Cette évolution serait sans effet dans un monde où la totalité de l'investissement serait assumée par la collectivité, sans contribution des usagers. Mais la multiplication des PPP, a conduit à mieux identifier le dénominateur pertinent, qui n'est pas le montant d'investissement, mais la subvention publique. C'est bien, en effet, la contrainte sous laquelle il s'agit d'optimiser le rendement social.

\section{Conclusion}

Au total, on aperçoit que ni l'apparition des partenariats public-privé dans le secteur des transports, ni la généralisation du cofinancement usager-contribuable, ne sauraient impliquer un simple repli sur une logique de la seule rentabilité financière. Mais on voit aussi que le critère de la valeur actualisée nette par euro investi doit être amendé en ne retenant au dénominateur que la part financée par le contribuable, c'est à dire l'euro public investi.

Toutefois, les vertus du critère peuvent être discutées lorsqu'on relâche l'hypothèse de constance de la VAN et du besoin de subvention dans le temps (Maurice 2005, Roy 2005). Et de toute évidence il sera d'autant plus pertinent qu'il sera associé au critère du taux de rentabilité immédiate. La procédure de mise en concurrence des projets sur la base de leur $V A N_{e} / S$ ne devient, en effet, pertinente que pour des projets dont la rentabilité immédiate atteint ou dépasse le taux d'actualisation.

Notons, enfin, que le type d'exploration des programmes que nous avons utilisé n'a pas pour seul mérite de confirmer un critère simple d'optimisation. Il permet aussi d'introduire dans la notion de programme de projets toutes sortes d'éléments de dynamisation: les caractéristiques socio-économiques des projets selon les dates de mise en service; des différenciations dynamiques de ces caractéristiques; la prise en compte des effets de complémentarité ou de substitution entre projets selon les ordres de leur réalisation; etc. La difficulté de mise en œuvre des simulations de programmes concerne alors les sources statistiques, les évaluations disponibles ne prenant que très rarement en compte ces éléments ${ }^{30}$.

Pour autant que l'on suppose résolue cette difficulté, le critère du ratio optimal $\left(\mathrm{VAN}_{\mathrm{e}}\right.$ / euro de financement public) n'est pas réductible à une seule série chronologique par projet. Celle-ci peut, en effet, varier de manière discontinue selon la place du projet dans un programme et selon les autres éléments du programme. Il ne reste bien alors que le recours aux simulations numériques pour déterminer la programmation optimale et c'est 
probablement ce qui en fait leur intérêt par rapport à la seule approche mathématique de l'optimisation. 


\section{Bibliographie}

ABRAHAM C. \& LAURE A. (1959) "Etude des programmes d'investissement routiers », Annales des Ponts et Chaussées, novembre-décembre.

BABUSIAUX D. (1990), Décision d'investissement et calcul économique dans l'entreprise, Economica / Technip, coll. Economie et statistiques avancées.

BAUMOL W. \& QUANDT R. (1965), «Investment and Discount Rates Under capital Rationing, A Programming Approach », The Economic Journal, 75(298), pp.317-329.

BERTONECHE M. \& LANGOHR H. (1977), "Le choix des investissements en situation de rationnement de capital. Comparaison des solutions fournies par différents modèles théoriques», Revue Economique, 28(5).

BLOY E., BONNAFOUS A, CUSSET J-M \& GERARDIN B. (1976), Evaluer la politique des transports, Economica / Presse Universitaires de Lyon, coll. « Economie publique de l'aménagement et des transports ».

BONNAFOUS A. \& JENSEN P. (2005), « Ranking Transport Projects by their Socio-economic Value or Financial Interest Rate of Return? », Transport Policy, 12, pp.131-136.

BONNAFOUS A. \& JENSEN P. (2006), «Le cofinancement usager-contribuable et le partenariat public-privé changent les termes de l'évaluation des programmes d'investissement public », Economie et Prévision, à paraître.

BONNAFOUS A. (1999), «Infrastructures publiques et financement privé : le paradoxe de la rentabilité financière », Revue d'Economie Financière, 51.

BONNAFOUS A. (2002), «Les infrastructures de transport et la logique financière du partenariat public-privé : quelques paradoxes », Revue Française d'Economie, 17(1).

BONNAFOUS A., JENSEN P. (2005). «Ranking transport projects by their socioeconomic value or financial internal rate of return?", Transport Policy, 12(2).

BONNEL P. (2004), Prévoir la demande de transport, Presses de l'école nationale des ponts et chaussées, Paris.

BRUNEL J. (2003), «L'influence de l'ordre de réalisation des infrastructures de transport: une simulation », document de travail du LET, en ligne sur : http://halshs.ccsd.cnrs.fr/halshs-00003980.

BUDIN K.J. et THOMPSON L.S. (2001), « Directions of Railway Reform », Rail International Schienen der Welt, sept.-oct., pp. 30-45.

COMMISSARIAT GENERAL DU PLAN (1993), Transports : pour une cohérence stratégique, Rapport de l'atelier présidé par Alain Bonnafous, CGP.

COMMISSARIAT GENERAL DU PLAN (1994), Transports: pour un meilleur choix des investissements, M. Boiteux (prés.), Paris : La Documentation Française.

COMMISSARIAT GENERAL DU PLAN (1996), Transports: pour un meilleur choix des investissements, M. Boiteux (prés.), Paris : La Documentation Française.

COMMISSARIAT GENERAL DU PLAN (1997), Transports : le prix d'une stratégie, A. Bonnafous (prés.), 2 vol., Paris : La Documentation Française.

COMMISSARIAT GENERAL DU PLAN (2001), Transports : choix des investissements et coût des nuisances, M. Boiteux (prés.), Paris : La Documentation Française.

COMMISSARIAT GENERAL DU PLAN (2005), Révision $d u$ taux d'actualisation des investissements publics, D. Lebègue (prés.), Paris : La Documentation Française.

EVERETT H. (1963), «Generalized Lagrange multiplier method for solving problems of optimum allocation of resources », Operations Research, 11(3) pp. 399-417. 
FAIVRE D’ARCIER B. et MIGNOT D. (1998), «Using Economic Calculation as a Simulation Tool to Assess Transport Investments », $8^{\text {th }}$ WCTR, Anvers.

HAYASHI Y. \& MORISUGI H. (2000), «International comparison of background concept and methodology of transportation project appraisal », Transport Policy, 7(1), pp.73-88.

LANGLOIS G. \& MOLLET M. (1999), Gestion financière, Fouchet, DECF, $2^{\mathrm{e}}$ édition.

LESOURNE J. (1972), Le calcul économique : théorie et application, Dunod, 459 p.

LORIE J.H. \& SAVAGE L.J. (1955), «Three problems in rationing capital », Journal of Business, 28(4), pp.229-239.

MAURICE J. (2005), "Classement des projets et choix public», Séminaire sur les dimensions critiques du calcul économique, Lyon, 13-14 septembre.

MOLNAR E. (2003), « Trends in Transport Investment Funding », Magnificent Seven Papers, ECMT.

QUINET E. (1990), Analyse Economique des transports, PUF, Paris

QUINET E. (1998), Principes d'économie des transports, Economica, Paris

QUINET E. (2000), «Evaluation methodologies of transportation projects in France », Transport Policy, 7(1), pp.27-34

ROTHENGATTER W. (2000), "Evaluation of infrastructure investments in Germany », Transport Policy, 7(1), pp.17-26

ROY W. (2005), «Evaluation des programmes d'infrastructure : Ordre optimal de réalisation sous contrainte financière ", Document de travail du LET, en ligne sur : http://halshs.ccsd.cnrs.fr/halshs00003971 .

VAN LAARHOVEN P. et AARTS E.H.L. (1992), Simulated Annealing: Theory and Applications, Dordrecht : Kluwer.

VERNIMMEN P. (2005), Finance d'entreprise, $6^{\mathrm{e}}$ édition par P. Quiry et Y. Le Fur, Dalloz.

VICKERMAN R. (2000), «Evaluation methodologies for transport projects in the United Kingdom », Transport Policy, 7(1), pp.7-16

WEINGARTNER M. (1963), Mathematical Programming and the Analysis of Capital Budgeting Problems, Englewood Cliffs: Prentice-Hall (reissued, London: Kershaw, 1974). 
AnNeXe: Caractéristiques économiques des projets autoroutiers utilisés dans les programmes simulés

$\begin{array}{lccc}\text { LIAISONS } & \text { TRE (1) } & \text { TRI } & \text { COÜT } \\ \text { Arles - Salon } & 74 \% & 7,50 \% & 283 \\ \text { Isle Adam - Amiens } & 45 \% & 6,10 \% & 488 \\ \text { Toulouse Pamiers } & 30 \% & 4,10 \% & 450 \\ \text { Tours - Alençon } & 28 \% & 3,60 \% & 698 \\ \text { Dôle - Bourg } & 20 \% & 7,10 \% & 737 \\ \text { Saintes - Rochefort } & 20 \% & 2,30 \% & 290 \\ \text { Tours - Vierzon } & 19 \% & 4,10 \% & 800 \\ \text { Annemasse-Thonon } & 18 \% & 7,90 \% & 378 \\ \text { Grenoble - Sisteron } & 17 \% & 2,50 \% & 1880 \\ \text { Sens - Courtenay } & 15 \% & 10,10 \% & 197 \\ \text { Orléans - Courtenay } & 13 \% & 3,00 \% & 470 \\ \text { Dijon - Dôle } & 12 \% & 9,50 \% & 213 \\ \text { Lyon - Balbigny } & 11 \% & 1,20 \% & 770 \\ \text { Ambérieu - Bourgoin } & 11 \% & 1,20 \% & 500 \\ \text { Rouen - Alençon } & 9 \% & 1,40 \% & 580 \\ \text { A88 Caen - Argentan } & 9 \% & 1,20 \% & 250 \\ \text { Troyes - Auxerre } & 4 \% & 0,90 \% & 1350\end{array}$

(1) Lorsque les taux de rentabilité immédiate étaient seuls disponibles pour les 17 projets, les TRE utilisés pour nos simulations ont été estimés à l'aide du modèle présenté dans la sous-section 2.1.

Source : Julien Brunel (2003) d'après Commissariat général du Plan (1993). Les coûts sont en millions d'euros.

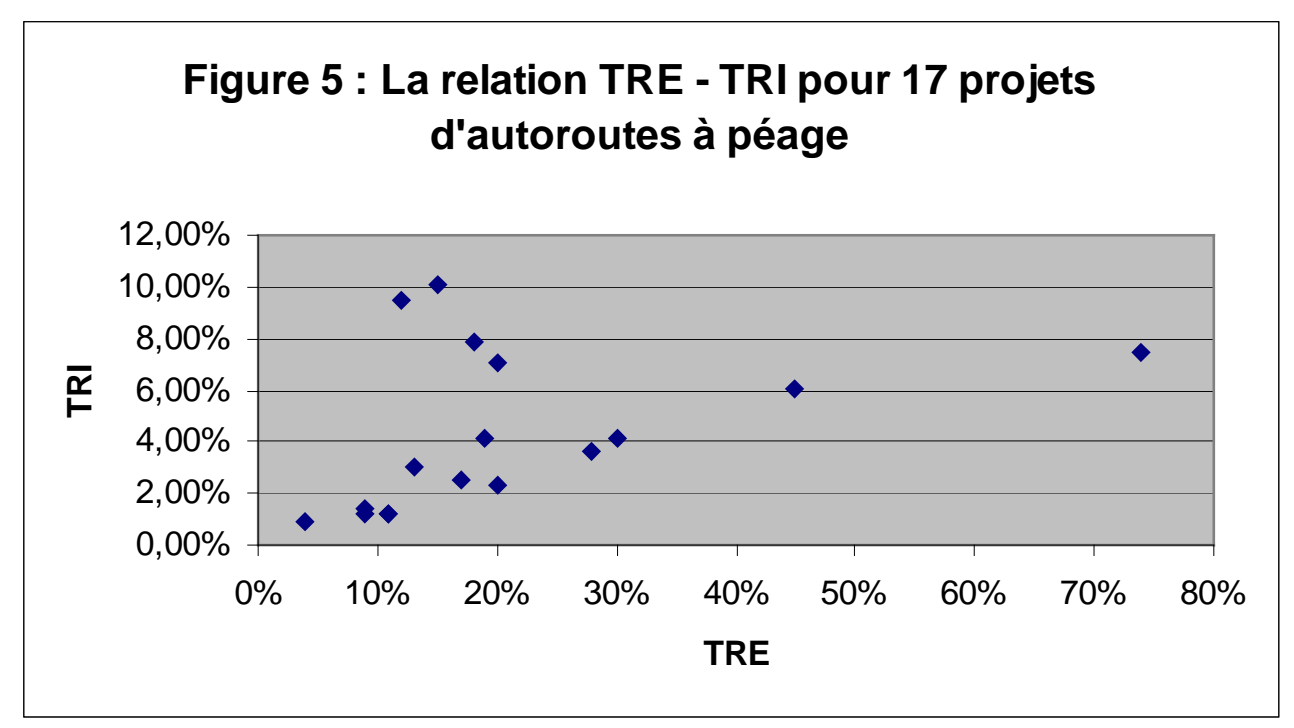


${ }^{1}$ Loi d'Orientation des Transports Intérieurs (LOTI) n82-1153 du 30 décembre 1982, Titre I - Chapitre III « Des infrastructures, équipements, matériels et technologies », article 14 (modifié par la loi du 25 juin 1999).

2 «Il faut bien reconnaître qu'on se heurte souvent à un certain scepticisme à l'égard des calculs économiques en général : est-il réaliste, se demandent certains, de vouloir codifier des décisions qui requièrent la prise en considération de multiples éléments de valeur très différente et dont beaucoup sont purement subjectifs ? (...) Il n'en reste pas moins que l'analyse de la rentabilité constitue, dans la mise en balance des avantages et des inconvénients que toute décision implique, un élément d'information irremplaçable qu'on a pas le droit de négliger » (p. 736-737, Abraham \& Laure 1959).

${ }^{3}$ Cette référence est choisie plutôt que d'autres plus anciennes car elle contient la première démonstration théorique de l'intuition de Jules Dupuit selon laquelle le surplus de l'usager est une composante de la variation d'utilité collective (évaluée par le surplus global de productivité).

${ }^{4}$ La première circulaire convenablement achevée du point de vue méthodologique a été celle de janvier 1970.

${ }^{5}$ Il s'agit de l'excédent brut d'exploitation et des avantages monétarisés (en pratique, ce sont surtout des gains de temps).

${ }^{6}$ Un groupe de travail du Plan, présidé par Marcel Boiteux, a proposé une première mise à jour de ces méthodes de calcul et des valeurs unitaires à prendre en compte pour les externalités en 1994. Un deuxième rapport plus complet et actualisé a été établi en 2001 (CGP, 1994 et CGP, 2001).

${ }^{7}$ Ce « seuil », ou taux d'actualisation du Plan, a été ramené de $10 \%$ à $8 \%$ au début des années 80 et n'a pas été révisé depuis. Cela a eu évidemment pour effet d'allonger sensiblement la liste de projets réputés rentables pour la collectivité. Mais cela n'a pas empêché que le poids des dépenses d'infrastructure dans le PIB soit, dans le même temps, diminué (Commissariat général du Plan, 1993). Le Commissariat Général au Plan (CGP 2005) a récemment révisé le taux d'actualisation officiel. D'un taux de $8 \%$ agrégeant un ensemble d'éléments mal définis, nous passons à un taux de $4 \%$ «nu». Les risques devront être clairement explicités et pris en compte par ailleurs pour chaque projet. Ce taux pourra décroître jusqu'à $2 \%$ à partir de 30 ans. La mise en œuvre de ce nouveau taux risque de conduire à une augmentation du nombre des évaluations établissant une valeur actualisée nette socio-économique positive.

${ }^{8}$ Avec une VAN positive

${ }^{9}$ Pour s'assurer que cet extremum est un maximum, il faut $d A\left(t^{*}\right) / d t \geq 0$, c'est à dire que les avantages (non actualisés) du projet soient non décroissants.

${ }^{10}$ La méthode utilisée pour arrêter le programme du $5^{\text {ème }}$ Plan était par exemple de classer les investissements par taux de rentabilité immédiate décroissant, et de s'arrêter quand on avait épuisé la contrainte budgétaire, ce qui revenait à appliquer ce principe.

${ }^{11}$ Réseau Ferré de France.

12 Entre 1956 et 1963, naissent cinq sociétés d'économie mixte concessionnaires d'autoroutes (SEMCA). Elles ne comportaient, en réalité, que des capitaux publics, mais leur relatif succès a conduit l'Etat à attribuer des concessions au secteur privé. Quatre sociétés ont ainsi été créées entre 1970 et 1973, dont les actionnaires étaient des banques et des entreprises de travaux publics. Le succès de ce début de privatisation sera compromis par les effets du choc pétrolier de 1973 et seule COFIROUTE survivra comme société privée. AREA sera transformée en SEMCA ; APPEL et ACOBA seront absorbées par deux autres SEMCA.

${ }^{13}$ Notons que le $8 \%$ du Plan est un seuil de TRE alors que le $8 \%$ garanti à la SNCF en taux réel et à RFF en taux nominal sont des TRI et que l'égalité entre ces valeurs est fortuite.

${ }^{14}$ Les calculs qui suivent se transposent sans difficulté avec une fonction exponentielle et les analyses qui en résultent n'en sont pas radicalement modifiées.

${ }^{15}$ Les détails des calculs sont présentés dans la présentation initiale de cette formalisation (Bonnafous, 2002).

${ }^{16}$ Les résultats présentés dans ce paragraphe résultent de simulations réalisées dans le cadre d'un séminaire de recherche du LET (Brunel, 2003). C'est également dans le cadre de ce séminaire dirigé par Alain Bonnafous qu'ont été réalisées par Pablo Jensen les premières simulations numériques qui devaient déboucher sur les résultats présentés dans le paragraphe 3.2.

${ }^{17} \mathrm{La}$ liste de ces projets est présentée en annexe. Il s'agit des projets du réseau français qui étaient en concurrence au début des années 90. Plusieurs ont été réalisés depuis avec une faible contribution apparente des finances publiques car ils ont été confiés à des sociétés d'autoroute qui ont pu utiliser le mécanisme d'adossement (cf. section 1).

${ }^{18}$ Une telle démonstration a cependant été tout récemment proposée par William Roy (2005) dans le cadre d'un travail mené avec les auteurs de cet article sur la problématique d'un programme optimal de projets.

${ }^{19}$ Dans cet article cité en référence, le résultat correspondant à celui qui est présenté sur la figure 4 ci-après est légèrement différent en raison d'une faiblesse de l'algorithme de « remplissage » de la contrainte financière qui a été corrigé entre temps.

${ }^{20}$ Critère selon lequel la date optimale de mise en service d'un projet, dès lors que son avantage net ne décroît pas avec le temps, est la première année pour laquelle son taux de rentabilité immédiate est au moins égal au taux d'actualisation.

${ }^{21}$ Bertonëche \& Langohr (1977) offrent une intéressante mise en perspective de ces travaux fondateurs, et Babusiaux (1990) une synthèse sur les modèles de programmation linéaire qui ont suivit.

${ }^{22}$ Notons que dans ce modèle simple, les VAN ne sont pas supposées variables au cours du temps. Pour un modèle plus général, mais aussi plus complexe, voir Maurice (2005) ou Roy (2005).

${ }^{23}$ Il est important de ne pas assimiler ce coût d'opportunité au coût social des fonds publics. Le coût social des fonds publics (shadow cost of public funds) est formé par les coûts de la collecte de l'impôt et les distorsions de prix associés.

${ }^{24}$ Le « coefficient d'utilité » dans la terminologie d'Abraham \& Laure (1959, p743)

${ }^{25}$ « This produce in effect a rank list of projects according to the Benefit-Cost Ratio (BCR). Which of these projects would be selected would then depend on an overall cash limit of finance for roads » (Vickerman 2000, p.8).

${ }_{26}^{26}$ "The benefit-cost criterion is used for setting priorities for the projects under evaluation» (Rothengatter 2000, p.22)

${ }^{27}$ Entreprises qui n'ont été privatisées qu'en 2005.

${ }^{28}$ voir par exemple Langlois \& Mollet (1999), ou Vernimmen (2005) 
${ }^{29}$ Bien entendu, ce critère s'applique après à la date optimale de mise en service du projet

${ }^{30}$ Cet exercice est cependant en cours dans le cadre du programme de recherche du LET. 